\title{
Precision $W$-pair physics with the YFSWW3 and KoralW Monte Carlos
}

\author{
M. Skrzypek ${ }^{a *}$, S. Jadach ${ }^{b}$, W. Płaczek ${ }^{c}$, B.F.L. Ward ${ }^{\mathrm{d}}$ and Z. Wạs ${ }^{\mathrm{e}}$ \\ ${ }^{a}$ Institute of Nuclear Physics, ul. Radzikowskiego 152, 31-342 Cracow, Poland \\ and CERN, TH Division, CH-1211 Geneva 23, Switzerland \\ b Institute of Nuclear Physics, ul. Radzikowskiego 152, 31-342 Cracow, Poland \\ ${ }^{\mathrm{c}}$ Institute of Computer Science, Jagellonian University, ul. Nawojki 11, 30-072 Cracow, Poland \\ ${ }^{\mathrm{d}}$ Department of Physics and Astronomy, The University of Tennessee, Knoxville, TN 37996-1200, USA \\ eInstitute of Nuclear Physics, ul. Radzikowskiego 152, 31-342 Cracow, Poland \\ and CERN, TH Division, CH-1211 Geneva 23, Switzerland
}

\begin{abstract}
We present the recent developments in the precision studies of $W$-pair and single- $W$ processes in $e^{+} e^{-}$collisions achieved with the help of the KoralW and YFSWW3 Monte Carlo generators. We focus on the theoretical precision of the measurements of $M_{W}$ and anomalous couplings on the example of $\lambda$ coupling. We present the mechanism of running these two independent codes in the form of one Concurrent Monte Carlo code. We describe also the extensions of KoralW necessary to emulate the kinematical region of single- $W$ process.
\end{abstract}

\section{CERN-TH/2002-261, UTHEP-02-0903, September 2002}

\section{Introduction}

In this note we give a short summary of our recent results and developments in the area of precision $W$-pair physics performed with the help of the Monte Carlo (MC) event generators KoralW [1-4] and YFSWW3 [5-9]. We will concentrate mostly on the LEP2 physics; however, some results for the energy range of future linear colliders will be also discussed.

The LEP2 data analysis is gradually approaching its final, highest precision stage [10]. The forecast accuracy of $1 \%$ for the $\mathrm{W}$-pair total cross section, $30 \mathrm{MeV}$ for $W$ mass or 0.01 for anomalous coupling $\lambda$ as an example of anomalous vectorboson triple-gauge couplings (TGCs), will be an impressive achievement. Also in the area of single- $W$ production the experimental precision for the total cross section is expected to be as high as $7 \%$.

Such a good experimental performance imme-

\footnotetext{
*Talk given by M. Skrzypek at 6th International Symposium on Radiative Corrections, 8-13 September 2002, Kloster Banz, Germany.
}

diately puts demands on the theoretical calculations - they should be accurate to at least half of the experimental precision. All kinds of theoretical corrections have been extensively studied throughout the last years and we will not attempt to cover this vast subject here. Any interested reader will find a review in the Reports $[11,12]$. We will only mention here that some of the most difficult corrections turned out to be the $\mathcal{O}(\alpha)$ electroweak $(\mathrm{EW})$ ones. These corrections have been included in the YFSWW3 code in the so-called "double-pole approximation" based on the expansion in the $\Gamma_{W} / M_{W}$ parameter and keeping only the highest (doubly) resonant contributions, all done in a gauge-invariant way [13]. Apart from YFSWW3, only one other MC code, the RacoonWW $[14,15]$, is able to calculate these corrections.

The available studies in the literature of theoretical precision have been restricted to the total cross section [12], lacking any discussion on the other basic observables such as $M_{W}$ or TGCs. The attempts to naively extrapolate the error of $\sigma_{W W}$ to the other observables (in the form of "er- 
ror bands" for example) have failed, as other observables do not depend so much on the overall normalization, but rather on the shape of some differential distributions. For that reason, we decided to study both $M_{W}$ [16] and TGCs [17] in detail. The TGC study has been done in collaboration with R. Brunelière, A. Denner, S. Dittmaier, S. Jézéquel, M. Roth and D. Wackeroth.

We have also done a number of upgrades and extensions of our MC codes. Among them the most important ones are: (1) the "merge without merging" of KoralW and YFSWW3 into a one MC code on an event-per-event basis, with the help of the novel technique of Concurrent Monte Carlo [4], and (2) the improvements in KoralW in the domain of single- $W$ processes [18], necessary to meet the experimental precision in this area.

\section{KoralW\&YFSWW3 - the Concurrent Monte Carlo for $W$ physics [4]}

We have developed two Monte Carlo codes for the $W$-pair physics: KoralW and YFSWW3. The first of them, KoralW, includes the complete, fully massive matrix element for all four-fermion processes, generated by the GRACE package [19] along with two independent and efficient multichannel algorithms for the four-fermion phasespace integration [20]. The YFSWW3 includes the complete $\mathcal{O}(\alpha)$ electroweak corrections to the $W$-pair production process in the double pole approximation, based on the on-shell calculations of Refs. [21,22]. Apart from that, the two programs share a number of features such as the initial state radiation (ISR) in Yennie-FrautschiSuura exponentiation up to $\mathcal{O}\left(\alpha^{3}\right)$ LL with multiple photons with non-zero transverse momenta, Coulomb correction or anomalous TGCs. Also the semi-analytical program KorWan for $W$-pair production and decay is included in both packages.

To meet the high precision requirements of the LEP experiments the corrections of both codes need to be available simultaneously, on an eventper-event basis. The most obvious way of achieving it, by merging the two codes at the level of source codes, is highly impractical. The already big codes (few hundred thousand lines) would become even larger; it would not be possible to maintain them separately, or the merging would have to be repeated after each new release. Therefore we proposed a different approach: the Concurrent Monte Carlo (CMC) [4]. In $\mathrm{CMC}$ both codes remain completely independent, but they run simultaneously and communicate in real time through the UNIX/LINUX facility called "named pipes" or "FIFO files". From the technical point of view, there is no need to modify any of the codes (apart from the changes necessary to accommodate the physics requirements) as the FIFO files behave just like ordinary ASCII files. A sample of such a setup is shown in Fig. 1, where we present the main operation mode

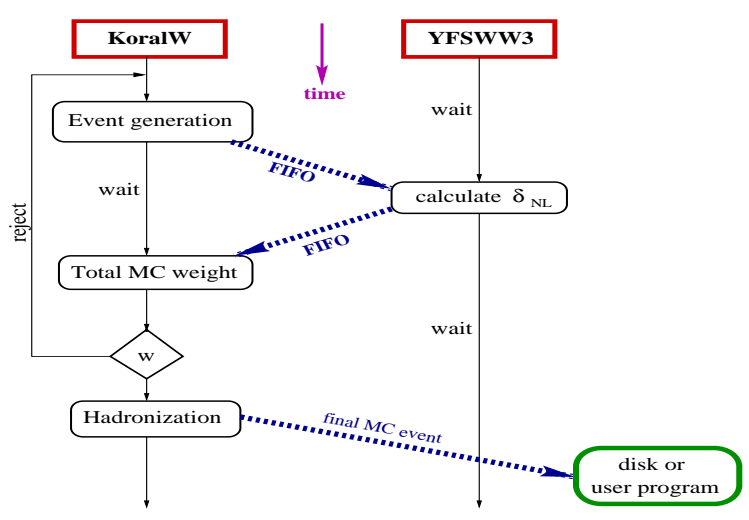

Figure 1. The time-flow of the CMC KoralW\&YFSWW3

of CMC KoralW\&YFSWW3 in which the events are constructed with full background four-fermion corrections and the $\mathcal{O}(\alpha)$ EW corrections.

The concept of CMC has a number of advantages: (1) it provides a natural mechanism of "encapsulation" for any codes written in any languages without any need to modify their source codes; (2) it has a "lego block" structure with many possible arrangements of involved codes (for example for reprocessing data by two KoralW codes running concurrently but initialized 
with different input parameters ); (3) it is naturally suited to use multiprocessor computers efficiently.

\section{Theoretical precision of $M_{W}$ measurement [16]}

How did we proceed with the estimation of the theoretical error of the $M_{W}$ measurement? We set-up a simplified framework based on onedimensional fits to the invariant mass of one of the $W$ 's. These fits were performed with the help of the semi-analytical code KorWan [2], based on the CC03 matrix element convoluted with the ISR and with the screened Coulomb correction, both matching exactly the corresponding corrections in KoralW and YFSWW3 MCs. In the first step, we checked that the fitting procedure works properly and we reproduce in the fit the value of the $W$ mass used in the $\mathrm{MC}$ simulation. This introductory exercise shows also that if one is interested only in the relative shifts of $M_{W}$ due to various effects, then any of the fitting functions (FFs) that we used is equally good.

In the next step we switched on and off various corrections in the MC sample and in FFs to see how they influence the $M_{W}$. We found out that the most complicated corrections: four-fermion background and EW non-leading $\mathcal{O}(\alpha)$ ones are both of the size of $1 \mathrm{MeV}$.

In the third step, we compared the results of the fits to the KoralW\&YFSWW3 data with the fits to the data generated by the RacoonWW MC code, taken from Ref. [23]. Both these codes include $\mathcal{O}(\alpha)$ EW and four-fermion corrections, but they differ in many aspects of the actual implementation of these corrections. Therefore such a comparison provides in particular a good test on the technical precision of the calculations. The fitted value of $M_{W}$ obtained from corresponding data from these two codes turned out to differ by less than $3 \mathrm{MeV}$.

Finally, we estimated the size of the possible missing corrections in KoralW\&YFSWW3. This has been done for missing parts of ISR $(<1$ $\mathrm{MeV}), \mathrm{FSR}(\sim 2 \mathrm{MeV})$ as well as non-factorizable $(<2 \mathrm{MeV})$ ones.

All the above work brought us to the fol- lowing conclusion: the electroweak theoretical precision of $M_{W}$ reconstruction through KoralW\&YFSWW3 at LEP2 energies is $\sim 5 \mathrm{MeV}$. This estimate includes a "safety factor" of 2 , due to the simplified 1-dimensional framework that we used. The above estimate is in good agreement with the small differences between the YFSWW3 and RacoonWW MCs. This value is well below the expected final LEP2 experimental precision of $\sim 30 \mathrm{MeV}$.

\section{Theoretical precision of anomalous $\lambda$ coupling measurement [17]}

This study of $\lambda$ coupling has been done in collaboration with R. Brunelière, A. Denner, S. Dittmaier, S. Jézéquel, M. Roth and D. Wackeroth.

The measurement of anomalous TGCs is primarily based on the distribution of the cosine of the $W$ boson production angle $\theta_{W}$. The most general parametrization of the anomalous TGCs requires fourteen complex parameters. In our analysis we concentrated on the simplest scenario of only one anomalous TGC, the $\lambda=\lambda_{\gamma}=\lambda_{Z}$. We adopted essentially the same strategy as for the $M_{W}$ analysis, i.e. we performed 1-dimensional fits to the $\cos \theta_{W}$ distributions.

As in the $M_{W}$ case, we began by a simple fit of the semi-analytical FF from KorWan. It turned out, however, that the size of the various effects obtained from such a fit differ significantly, depending on the acceptances applied to the MC data. Therefore we had to find a way of incorporating more realistic experimental acceptances into FFs. We did this by using the Monte Carlo Parametric Fitting Function (MPFF), constructed directly as a parametrization of MC data, in the form of a polynomial in $\lambda$ and $\cos \theta_{W}$. In particular we used the reallife ALEPH acceptances. The performed fits to MPFFs thus show that the $\mathcal{O}(\alpha)$ NL EW corrections lead to a $\lambda$ shift of about 0.01 and that the differences between KoralW\&YFSWW3 and RacoonWW are very small, below 0.001. In the next step we also looked at what happens when we extend our analysis to the different channels, other than $\mu \nu_{\mu} q q$. This has been done with 
the help of "transfer matrices", constructed on the basis of the full ALEPH detector simulation. These matrices have been applied to our $\mathrm{MC} \cos \theta_{W}$ distributions. As a result we showed that the effect of $\mathcal{O}(\alpha)$ NL EW corrections on $\lambda$ is similar (within 15\%) in all channels, including the difficult four-quark and tau-semileptonic ones. In the last step we have estimated the size of missing corrections in both MC codes.

As a result we came to the conclusion that the EW theoretical uncertainty in $\lambda$ of the KoralW\&YFSWW3 and RacoonWW MC codes is $\sim 0.005$. As before this number includes a "safety factor" of 2 due to the 1-dimensional fit and missing higher-order effects.

\section{Improvements of KoralW for the single- $W$ processes [18]}

Apart from the $W$-pair production, another interesting process involving the $W$ boson is the single- $W$ process. It is usually defined as the $e \bar{e} \rightarrow e \bar{\nu}_{e} f^{\prime} \bar{f}$ with outgoing $e$ close to the beam line (say, $\cos \theta_{e} \geq 0.95$ ) [23]. In such a configuration the $t$-channel graphs dominate. As a consequence, the photonic radiation based on initial state only, sufficient for $W$-pair production, has to be corrected to include the screening of the electric charge (ECS) due to the dipole-like structure formed by initial- and final-state electrons. This screening is responsible for the strong suppression of photons with large transverse momenta and for the change of scale of the "leading logarithms" from $s$ to $|t|$.

The KoralW code, based on ISR, has missed the ECS effect. Therefore we have proposed [18] a simple way of reintroducing it by just adding an additional weight, without any modification of the original code. The weight results from an educated guess rather than the rigorous derivation from the first principles. It is constructed as a ratio of one-photon differential distributions of a coherent sum of IS and FS radiation over an analogous incoherent IS and FS sum. These distributions are exact in the soft photon limit and have the simple form of $\left(p_{a} / k p_{a}-p_{b} / k p_{b}\right)^{2}$. In order to maintain the independence of the program of the dummy infra-red cut-off $\epsilon_{I R}$ we also introduced the corresponding virtual weight. As in the real-emission case, we have constructed it as an ansatz that fulfills the requirement of cancelling the spurious $\epsilon_{I R}$ dependence. The overall precision of this ECS ansatz we estimate at $2 \%$, because of some missing nonleading terms.

In order to meet the experimental requirements of LEP2, we had to introduce also the effect of running the QED coupling constant in the $e$ vertex from the $M_{W}^{2}$ to $t$ scale. We have done this in the form of an overall factor $\left(\alpha(t) / \alpha_{G_{\mu}}\right)^{2}$. Such a naive ansatz has a precision better than $2 \%$, see Ref. [24].

In summary, we estimate at $3 \%$ the overall precision of the proposed improvements in KoralW in the single- $W$ region of phase space. This is sufficient for the expected LEP2 experimental precision of about $7 \%$.

We present in Table 1 some numerical results for the single- $W$ total cross sections in the $e^{+} e^{-} \rightarrow e^{-} \bar{\nu}_{e} u \bar{d}$ process with the following cuts: $\cos \theta_{e} \geq 0.997, M_{q \bar{q}} \geq 45 \mathrm{GeV}$. As can be seen, the effects of ECS and running of QED coupling cancel each other in the total cross section in a wide energy range, from the LEP2 up to future linear colliders area. This cancellation is however purely accidental and would not be true for differential distributions.

\section{Summary}

In this short note we presented recent results and developments in precision $W$-pair physics related to the KoralW and YFSWW3 MC programs:

(1) We established that the theoretical precisions, so far omitted in the literature, of fundamental LEP2 observables other than total cross section are: $5 \mathrm{MeV}$ for $M_{W}$ (LEP2 experimental precision $\sim 30 \mathrm{MeV}$ ) and 0.005 for anomalous $\lambda$ coupling (LEP2 experimental precision $\sim 0.01$ ). These precisions fully meet the LEP2 experimental requirements.

(2) We proposed a new way of combining independent MC codes on an event-per-event basis, by running them in parallel and providing the realtime data exchange between them by means of the UNIX/LINUX facility of "named pipes". We 
Table 1

The total cross sections with ECS effect (ECS) and running QED coupling (RCC)

\begin{tabular}{lrrrr}
\hline $\mathrm{E}[\mathrm{GeV}]$ & $\sigma_{R C C}^{E C S}[\mathrm{fb}]$ & $1-\sigma_{R C C} / \sigma_{R C C}^{E C S}$ & $1-\sigma^{E C S} / \sigma_{R C C}^{E C S}$ & $1-\sigma / \sigma_{R C C}^{E C S}$ \\
\hline 190 & $87.11(22)$ & $5.65(15)$ & $-5.16(2)$ & $0.81(14)$ \\
200 & $103.60(26)$ & $5.49(15)$ & $-5.13(2)$ & $0.67(15)$ \\
500 & $807.5(2.7)$ & $4.92(21)$ & $-4.68(2)$ & $0.51(21)$ \\
\hline
\end{tabular}

used this scheme to create a Concurrent MC KoralW\&YFSWW3 that generates $W$-pair events with both four-fermion background and $\mathcal{O}(\alpha)$ corrections.

(3) We presented the upgrade of KoralW in the kinematical domain of single- $W$ targeted at better emulation of the $t$-channel-dominated photonic radiation. Thanks to this upgrade, the overall precision of KoralW for this type of processes at LEP2 has become $3 \%$ (LEP2 experimental precision $\sim 7 \%$ ).

\section{Acknowledgements}

This work has been supported in part by the Polish Government grants KBN 5P03B09320, 2P03B00122, the European Commission 5-th framework contract HPRN-CT-2000-00149, the NATO Grant PST.CLG.977751, the US DoE contract DE-FG05-91ER40627 and the PolishFrench Collaboration within IN2P3 through LAPP Annecy.

\section{REFERENCES}

1. M. Skrzypek, S. Jadach, W. Płaczek and Z. Wạs, Comput. Phys. Commun. 94, 216 (1996).

2. M. Skrzypek et al., Phys. Lett. B372, 289 (1996).

3. S. Jadach et al., Comput. Phys. Commun. 119, 272 (1999).

4. S. Jadach et al., Comput. Phys. Commun. 140, 475 (2001).

5. S. Jadach, W. Płaczek, M. Skrzypek and B. F. L. Ward, Phys. Rev. D54, 5434 (1996).

6. S. Jadach et al., Phys. Lett. B417, 326 (1998).

7. S. Jadach et al., Phys. Rev. D61, 113010 (2000).
8. S. Jadach et al., Phys. Rev. D65, 093010 (2002).

9. S. Jadach et al., Comput. Phys. Commun. 140, 432 (2001).

10. R. Chierici, these Proceedings.

11. Physics at LEP2, edited by G. Altarelli, T. Sjöstrand and F. Zwirner (CERN 96-01, Geneva, 1996), 2 vols.

12. Reports of the Working Groups on Precision Calculations for LEP2 Physics, edited by S. Jadach, G. Passarino and R. Pittau (CERN 2000-009, Geneva, 2000).

13. R. Stuart, Nucl. Phys. B498, 28 (1997), and references therein.

14. A. Denner, S. Dittmaier, M. Roth and D. Wackeroth, Nucl. Phys. B587, 67 (2000).

15. A. Denner, S. Dittmaier, M. Roth and D. Wackeroth, Nucl. Phys. Proc. Suppl. 89, 100 (2000).

16. S. Jadach et al., Phys. Lett. B523, 117 (2001).

17. R. Brunelière et al., Phys. Lett. B533, 75 (2002).

18. S. Jadach et al., Electric charge screening effect in single-W production with the KoralW Monte Carlo, hep-ph/0209268.

19. J. Fujimoto et al., GRACE User's manual, version 2.0, MINAMI-TATEYA collaboration.

20. M. Skrzypek and Z. Wąs, Comput. Phys. Commun. 125, 8 (2000).

21. J. Fleischer, F. Jegerlehner and M. Zrałek, Z. Phys. C42, 409 (1989).

22. K. Kołodziej and M. Zrałek, Phys. Rev. D43, 3619 (1991).

23. M. Grünewald et al., Four-fermion production in electron-positron collisions, in Ref. [12], p. 1.

24. G. Passarino, Nucl. Phys. B578, 3 (2000). 